\section{Argumentación y construcción de validez en la Planificación Estratégica-Situacional de Matus}

\author{
Argumentation and construction of validity in \\ Carlos Matus' Situational Strategic Planning
}

Francisco Javier Uribe Rivera 1

\section{Introducción}

1 Escola Nacional de Saúde Pública Sergio Arouca, Fundação Oswaldo Cruz, Río de Janeiro, Brasil.

Correspondencia F. J. U. Rivera

Departamento de Administração e Planejamento em Saúde, Escola Nacional de Saúde Pública Sergio Arouca, Fundação Oswaldo Cruz. Rua Leopoldo Bulhões 1480,

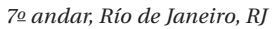
21041-210, Brasil. uribe@ensp.fiocruz.br

\begin{abstract}
This study analyzes the process of producing a
Abstract situational plan according to a benchmark from the philosophy of language and argumentation theory. The basic approach used in the analysis was developed by Carlos Matus. Specifically, the study seeks to identify the inherent argumentative structure and patterns in the situational explanation and regulatory design in a plan's operations, taking argumentative approaches from pragma-dialectics and informal logic as the analytical parameters. The explanation of a health problem is used to illustrate the study. Methodologically, the study is based on the existing literature on the subject and case analyses. The study concludes with the proposition that the use of the specific references means introducing greater rigor into both the analysis of the validity of causal arguments and the design of proposals for interventions, in order for them to be more conclusive in achieving a plan's objectives.
\end{abstract}

Strategic Planning; Health Planning; Planning Techniques
El objetivo de este trabajo es analizar en qué medida la Planificación Estratégica-Situacional (PES), aplicada al campo de salud, puede ser redefinida a partir de un enfoque comunicativoargumental. Se trata en el fondo de identificar y reforzar un componente que está presente en el PES, pero que a veces se oculta tras una representación más estratégica y metodológica. La relevancia del trabajo está vinculada al objetivo de introducir un mayor rigor lógico-discursivo en el proceso de producción de un plan, y de potenciar su base de legitimidad.

El PES no será problematizado en lo que se refiere a sus puntos fuertes y débiles. Este análisis crítico ya fue suficientemente desarrollado y consolidado en el trabajo de Rivera ${ }^{1}$. Ahora bien, este enfoque se convirtió en una propuesta ampliamente reconocida en América Latina y, específicamente, en Brasil, debido al intenso trabajo de difusión y formación realizado por su mentor ideológico, Carlos Matus, y por sus seguidores.

Matus, economista chileno, ex-ministro del presidente Salvador Allende, ganó un reconocimiento internacional por el trabajo de formulación y aplicación de un enfoque estratégico de planificación apoyado en una sólida matriz, donde podemos identificar rasgos de un enfoque comunicativo y argumental, que aquí pretendemos 
resaltar y explorar con el objeto de redimensionar su propuesta original. Concretamente, reconocemos las aportaciones de la Teoría de la Acción Comunicativa de Habermas 2 y de la Teoría de las Conversaciones de Flores 3 , que es una aplicación en el campo gerencial de la filosofía del lenguaje de Austin ${ }^{4}$ y Searle 5 . Asimismo, observamos en su obra referencias a la teoría argumentativa, aunque de manera más difusa. Por lo tanto, lo que nos parece que contribuye más a este trabajo se refiere a cómo esta teoría, especialmente la pragma-dialéctica de van Eemeren ${ }^{6}$ y la lógica informal, puede colaborar en la reconstrucción del PES, en el sentido anteriormente definido y por la relevancia que le atribuimos a este trabajo. Este sentido corresponde a una preocupación acerca de las condiciones de validez de las propuestas del plan, que podrían ser reforzadas argumentativamente.

El concepto de argumentación utilizado es el de Habermas: un tipo de acto de habla en el que los participantes transforman en tema las pretensiones de validez que se convierten en problemáticas y tratan de aceptarlas o rehusarlas mediante argumentos. Una argumentación contiene razones conectadas de forma sistemática con las pretensiones de validez de la emisión o manifestación problemáticas y su fuerza depende de la pertinencia de las razones 2 .

\section{La planificación situacional como planificación comunicativa}

Matus 7 contribuyó a introducir una visión comunicativa en la teoría de la planificación, dominada hasta entonces por un tipo de paradigma economicista, tecnocrático y divorciado de la política, calificado como paradigma normativo. Este autor es uno de los responsables por una reconceptualización de la planificación que pasó a ser entendida como un proceso eminentemente interactivo, superando así la concepción de la misma como enfoque de un actor único, a saber el Estado, operando dentro de contextos dominados por el determinismo (o por reglas objetivas y permanentes).

La puesta en práctica de una visión interactiva implicó la necesidad del análisis de viabilidad política, como algo inmanente a la planificación, redundando en la consideración de todos los actores sociales y políticos involucrados en un plan. Sin embargo, al mismo tiempo, esta visión llevó a la superficie el componente comunicacional de los planes, de la búsqueda de la construcción de la legitimidad en procesos cooperativos de diálogo, pieza esencial de la posibilidad de viabilizar un plan de acción.
La planificación estratégico-situacional es un enfoque de análisis de problemas que se constituye de cuatro momentos de cálculo:

- Momento explicativo: que consiste en la selección de los problemas estratégicos del plan, en su descripción y en su explicación causal, o sea, en la definición de las causas encadenadas que generan los descriptores de los problemas. Se entiende por descriptor un síntoma, una manifestación o la expresión directa de un problema. Por ejemplo, en el caso de una sobrecarga de un servicio de urgencia: un largo tiempo de espera y/o un número exagerado de personas atendidas por profesional por hora. Este momento culmina con la selección de los nudos críticos del modelo causal y con su descripción. Los nudos críticos están representados por causas de alto impacto sobre los descriptores de los problemas, sobre las cuales es posible actuar en términos prácticos y es oportuno políticamente intervenir.

- Momento normativo: que consiste en la definición del plan por operaciones, equivalentes a compromisos de acción, asumidos con vistas a enfrentar los nudos críticos. Junto a las operaciones, en este momento se definen los resultados esperados del plan y el grado de influencia del escenario sobre la realización de las operaciones.

- Momento estratégico: donde se realiza el análisis de viabilidad del plan a partir del análisis de los actores y se diseña, si es necesario, una estrategia para viabilizar las operaciones de posibilidad dudosa o crítica.

- Momento táctico-operacional: que es el momento de la implementación del plan, del monitoreo o evaluación y de la prestación de cuentas, que consiste en la entrega de información sobre el grado de cumplimiento de los objetivos y compromisos del plan, con la finalidad de corregir los rumbos del plan.

Para Matus 7, el plan tiene una estructura comunicativa, en la que se pueden distinguir, analíticamente hablando, un conjunto de actos de habla que a la luz de la filosofía del lenguaje, de Austin ${ }^{4}$, Searle ${ }^{5}$ y Searle \& Vanderveken ${ }^{8}$, permiten discernir cómo funcionan, a un cierto nivel, los momentos de la planificación. En el momento explicativo se destacan las afirmaciones o aserciones, las expresiones y las declaraciones (éstas en menor cantidad aquí). Mediante las afirmaciones se constatan hechos y procesos problemáticos, como, por ejemplo, que en una determinada ciudad existe una alta razón de mortalidad materna. Las afirmaciones pueden ser verdaderas o falsas y deben ser probadas. Mediante las expresiones se califican estos hechos usando frases adjetivas que aceptan, rechazan o jerarquizan las aserciones. Por ejemplo, nos felicitamos por la buena cobertura sanitaria 
alcanzada y lamentamos o condenamos el empeoramiento de los indicadores de mortalidad. A través de las declaraciones priorizamos problemas y elegimos nudos críticos cuando afirmamos que, dentro de ciertos límites, el problema de la diarrea infantil es más importante que la tuberculosis, o que la principal causa de la baja resolución de la atención primaria es la insuficiente infraestructura de unidades de salud. En estos casos se generan situaciones nuevas, puesto que se decide actuar sobre estos problemas en el sentido de su superación. En el momento normativo se destacan los actos compromisorios (compromisos de acción), las directrices y declaraciones. A través de los compromisos asumimos la promesa de efectuar una acción. A través de las directrices ordenamos a determinados actores que efectúen algunas acciones; y a través de las declaraciones indicamos responsables de la realización de módulos de acción. El momento táctico-operacional es un momento donde predominan las aserciones del proceso de evaluación de los compromisos. Para Matus, los productos terminales de los planes son las operaciones como compromisos de acción. Las aserciones y expresiones son productos intermediarios que contribuyen en la formulación de los compromisos.

Las operaciones deben estar bien organizadas en términos lingüísticos. No pueden ser formuladas como objetivos vagos, criterios genéricos de acción o simples recomendaciones. Las operaciones deben ser enunciadas de forma sintética y expresar una propuesta de intervención, a través de verbos que expresen acciones concretas. Por ejemplo, sensibilizar a la población no indica una acción concreta, sino que es el resultado de una acción anterior como realizar campaña educativa. Para que las operaciones denoten compromisos de acción, sus productos (bienes y servicios inmediatamente generados) y resultados (efectos de los productos) deben también ser enunciados de manera objetiva, articulando indicadores cualitativos e/o cuantitativos que puedan ser mensurados. Finalmente, las operaciones como compromisos deben articular lingüísticamente responsables y tiempos de realización.

Uno de los mayores problemas del cálculo gerencial es la dificultad para formular lingüísticamente las operaciones, correspondiendo esta dificultad a una incompetencia directiva genérica que crea serios puntos muertos en el avance de las proposiciones de modificación de la realidad.

La aplicación de la teoría de los actos de habla en el campo de la planificación puede tener varios usos prácticos:

- Evaluación de la proporción de los actos de habla en la estructura comunicativa de síntesis y, especialmente, de los compromisos de acción, que deberían predominar;

- Evaluación del grado de precisión de los compromisos de acción;

- Evaluación del grado de coherencia existente entre afirmaciones y expresiones, por un lado, y compromisos de acción, por otro;

- Análisis del conjunto de módulos específicos del plan por operaciones, o sea de los componentes internos de los compromisos (acciones, sub-acciones, actividades), de modo que se verifique si estos cubren sin duplicaciones ambiguas el universo de la acción comprometida;

- Seguimiento longitudinal de los actos de habla, para verificar en qué medida los resultados constatados (aserciones) corresponden al cumplimiento de los compromisos; en qué medida las nuevas aserciones (por ejemplo, las afirmaciones que representan el nuevo estado de los descriptores) revelan la persistencia o la superación de los hechos problemáticos constatados anteriormente; y en qué medida los nuevos compromisos propuestos a partir de las rendiciones de cuentas sobre el alcance del plan revelan la continuidad, el reajuste o la superación de los compromisos anteriores.

A partir de una aplicación de la Teoría de la Acción Comunicativa, que se apoya en el enfoque pragmático de Austin y Searle, Rivera 1,9, se elabora una comprensión del plan como un conjunto de pretensiones de validez, cuyo valor de veracidad, corrección y autenticidad depende de la implementación de discursos argumentativos amplios que permitan la participación y el desarrollo de la capacidad de aceptación del mayor número de afectados. Los objetivos centrales del plan por operaciones o compromisos de acción pueden ser interpretados en el contexto de la salud pública como formas de regulación social sobre el destino de los productos del sistema administrativo de salud y de la previsión social. Este proceso de distribución de bienes y servicios sociales representa la aplicación de normas y valores vinculados al valor de la vida humana de los grupos poblacionales, y se relaciona con las necesidades sociales identificadas, con los niveles de inclusión y el grado de distribución de los beneficios sociales esperados. Dicho de otra forma, esa definición tiene todos los visos de un discurso práctico 2 para fijar normas específicas, que representan oportunidades para la satisfacción de necesidades sociales. Su legitimidad depende del ejercicio de la descentralización y participación de los ciudadanos. La validez de esas normas no puede ser evaluada por el criterio de veracidad, pero sí por el criterio de corrección o justicia. Matus 7 afirma que el plan es una oferta para enfrentar problemas y que si esta oferta es 
aceptada por la base (que tiene legitimidad para ello) se transforma en compromiso. Esto refuerza nuestra visión del plan como pretensiones que deben ser rescatadas en procesos comunicacionales amplios, lo más correctos posibles en términos procedimentales, con capacidad de generar consensos.

Por otro lado, autores como Matus 7,10,11, Rivera \& Artmann 8,12 , Pitta \& Rivera 13 subrayan la existencia de un componente argumentativo en el proceso de formulación y de validación del plan situacional. Estos autores sostienen de manera directa o indirecta que el plan es una apuesta argumentativa, es decir, no se trata de un cálculo técnico-científico marcado por la certeza, sino de un cálculo técnico-político de futuro que evita el componente predictivo en nombre de la previsión, que implica trabajar con varias posibilidades. De esta forma, un plan es una posibilidad entre otras, cuya consistencia depende de la fuerza de los argumentos involucrados en el análisis de los problemas semi o "cuasi estructurados" 7,14,15 que le sirven de base, de los argumentos que expresan la definición de los descriptores, relaciones causales y nudos críticos de estos problemas, así como de los argumentos que ayudan a construir el escenario del plan, sus propuestas de intervención y sus posibilidades estratégicas.

Debido a su carácter semi o cuasi estructurado, los problemas de salud corresponden a situaciones de incertidumbre, que son aquellas en las que no se puede enumerar a priori todas las variables involucradas (en sus procesos causales y de intervención), y que sólo pueden ser tratadas a partir de modelos probabilísticos e intervenciones creativas. La problemática sectorial está fuertemente imbricada con otros ámbitos, como vivienda, educación, nivel de ingresos, hábitos de vida, cultura, etc., y es generada y contrastada en el contexto de una dinámica socio-política creativa, imaginativa, de difícil previsión ${ }^{15}$. En esta medida, planificar la intervención sobre problemas de salud implica un abordaje multisectorial y comunicacional, movilizando preferentemente todos los actores involucrados, para construir situaciones de consenso argumentativo, que permitan reducir la fuerte incertidumbre y generar propuestas dotadas de legitimidad.

Sostenemos que esa naturaleza peculiar de la problemática de salud (como parte de lo social) refuerza la idea del plan como apuesta argumentativa. Esta parece ser la intuición básica de Matus, cuando realiza la definición del plan como apuesta. Convencido de que este recurso de cálculo puede fallar, a pesar de la apertura hacia un modelo de previsión, creativo e estratégico, Matus afirma la idea de que al hacer un plan realizamos una apuesta no exenta de vulnerabilidades, siendo necesario lo que el llama análisis de vulnerabilidad y confiabilidad del plan, que correspondería a un análisis profundo de los argumentos que constituyen el plan. Con más precisión, Matus en Teoría del Juego Social 11 y el Plan como Apuesta 10 llama la atención sobre la necesidad de evaluar la solidez de las relaciones causales o de las argumentaciones causales y relaciona el análisis específico de vulnerabilidad y confiabilidad con el modelo de argumentación de Toulmin 16. Es esto lo que nos lleva en el punto siguiente a discutir el plan como argumento.

\section{Planificación situacional y argumento}

En el momento explicativo se puede ilustrar el carácter de apuesta argumentativa de un plan situacional. Por explicación situacional se entiende un recorte problemático de la realidad social, hecho con la finalidad de promover una acción. Este recorte explicativo debería obedecer a algunos requisitos: la explicación es autoreferencial, es siempre la explicación de un actor; es dinámica, articula pasado, presente y futuro; es policéntrica en el sentido de que debe tomar en cuenta la explicación del otro; es totalizadora, en el sentido de que debe incorporar todas las dimensiones de la realidad a las que pertenecen las variables causales del problema; es riguro$s a$, en el sentido de que es una explicación que supera las causas sintomáticas o el epifenómeno del problema y procura discernir causas más esenciales, etc.

Una de las principales características de la explicación situacional es el policentrismo, que responde en gran medida a la definición del enfoque respectivo como comunicativo. Matus 7 asume a partir de autores del enfoque hermenéutico como Ortega 17 , que la explicación situacional es un diálogo entre un actor y los otros actores involucrados en la generación y en el enfrentamiento de los problemas reconocidos. Este diálogo implica la necesidad de hacer patente la circunstancia del otro. Una lectura habermasiana de este diálogo sugiere la posibilidad de una fusión de horizontes, o sea la posibilidad de rebasar contextos particulares y abrirse a una construcción explicativa intersubjetiva, que no sería en absoluto la explicación de un único actor.

Entendemos por diálogo un intercambio lingüístico cooperativo entre dos o más personas, orientado a la consecución de un fin común 18. En el caso de una explicación el fin común del diálogo pertinente sería producir una explicación de consenso, incluyendo todos los componentes de su esquema operacional, esbozado 
anteriormente: descripción, selección de causas, encadenamiento de las mismas, selección de nudos críticos y descripción de los mismos. Cuando este diálogo es contradictorio, implicando la necesidad de resolver una diferencia de opinión entre un protagonista y un antagonista, este diálogo puede ser considerado un proceso dialéctico de argumentación o un diálogo crítico.

¿Cuál es el concepto de argumentación que preside el diálogo de la planificación? Matus 10 utilizaba los términos argumentos y causas como sinónimos. Esta distinción corresponde al uso implícito que algunos autores, como van Eemeren 6 , hacen del término argumento como la premisa o razón que justifica o explica un punto de vista. Otros autores utilizan el concepto de argumento como un sistema con dos partes, las premisas o razones, de un lado, y el punto de vista a ser defendido, de otro 18,19. Argumentar, de cualquier modo, sería defender un punto de vista a partir de razones 6,18. En el esquema de la explicación situacional, el enunciado del problema es un punto de vista que debe ser fundamentado en razones. Por otro lado, las causas generadoras del problema pueden ser consideradas puntos de vista que pueden ser justificados a partir de otros argumentos causales.

Una de las primeras definiciones del momento explicativo es la construcción del conjunto de descriptores (indicadores cuali/cuantitativos) del enunciado del problema, que recibe el nombre de vector de definición del problema (VDP). Para que el VDP tenga validez, cada uno de los descriptores debe ser necesario para precisar o calificar el enunciado del problema y el conjunto de los descriptores debe ser suficiente. Necesario es todo evento en cuya ausencia otro evento no puede ocurrir y suficiente es todo evento o conjunto en cuya presencia otro evento debe ocurrir 6,18.

Para el PES un vector suficiente quiere decir que si eliminamos las cargas negativas que los descriptores expresan, o sea si estos son modificados positivamente, el problema queda parcial o totalmente resuelto o superado. La pregunta por la suficiencia es crucial, pues este análisis puede llevar a la constatación de que un determinado vector no es completamente suficiente, sugiriendo que falta un descriptor (y, por tanto, un conjunto de causas vinculadas a él), lo que puede significar un plan truncado o parcialmente eficaz. Esta operación de descripción puede ser interpretada (a la luz de de la teoría argumentativa) como una justificación argumental del enunciado del problema, de tal manera que los descriptores pueden asemejarse a premisas que justifican el enunciado como conclusión. Como vimos, esta operación está influenciada, en pri- mer lugar, por el desafío lógico del análisis de necesidad y suficiencia.

Definidos los descriptores, cabe en seguida listar sus causas y conectar las causas entre sí, pues éstas se condicionan mutuamente, configurando un sistema de causalidad. Esta definición es la explicación propiamente dicha. A la luz de la teoría argumentativa 6,18,20 sostenemos la hipótesis de que la explicación situacional del PES tiene una estructura argumentativa que combina un tipo de argumento múltiple, coordinado y subordinado. Esta distinción corresponde al siguiente entendimiento: el argumento múltiple es un conjunto de argumentos independientes que justifican una tesis o enunciado; el argumento coordinado es un conjunto de argumentos interdependientes; y el argumento subordinado, es un conjunto de argumentos en que el nuevo argumento justifica el anterior transformado en punto de vista, en una línea o cadena vertical de causalidad o justificación. Para van Eemeren et al. 21, la estructura argumentativa es una opción del protagonista de un proceso crítico, dialéctico, de discusión. Una estructura coordinada puede convertirse en una necesidad cuando las objeciones actuales o anticipadas sugieren que un determinado argumento no es suficiente o concluyente para explicar un punto de vista o evento. Una estructura subordinada puede justificarse cuando determinados argumentos reciben objeciones fácticas o anticipadas, revelando una debilidad que sugiere la necesidad de otros argumentos que justifiquen o expliquen mejor a los primeros.

Pondremos un ejemplo de explicación situacional para ilustrar mejor lo que estamos diciendo (Figura 1). Se trata de una explicación sencilla producida por un grupo de trabajo de un curso de gestión y planificación en salud del estado del Ceará, Brasil. Este grupo procesó el problema de la alta mortalidad materna, con un único descriptor de síntesis: razón de mortalidad materna de 86,6/100.000 nacidos vivos para todo el Estado.

En este ejemplo hay un problema claro de capacidad de resolución de la atención al prenatal, que se expresa por una alta mortalidad materna. Lo importante aquí es que el proceso de causalidad está conformado por tres grandes líneas o cadenas causales y que estas líneas actúan de manera conjunta, en el sentido de generar un impacto sobre el problema. Las líneas son:

- Línea de la baja calidad de la atención primaria en el prenatal, cuyo principal descriptor es el elevado porcentaje de embarazo de riesgo no diagnosticado (NC1.1);

- Línea de deficiencia en la asistencia hospitalaria al parto y puerperio, cuyos descriptores 
Figura 1

Explicación situacional de la alta razón de mortalidad materna en el Estado de Ceará, Brasil.

Diagrama de flujo situacional

Causas

Descritor

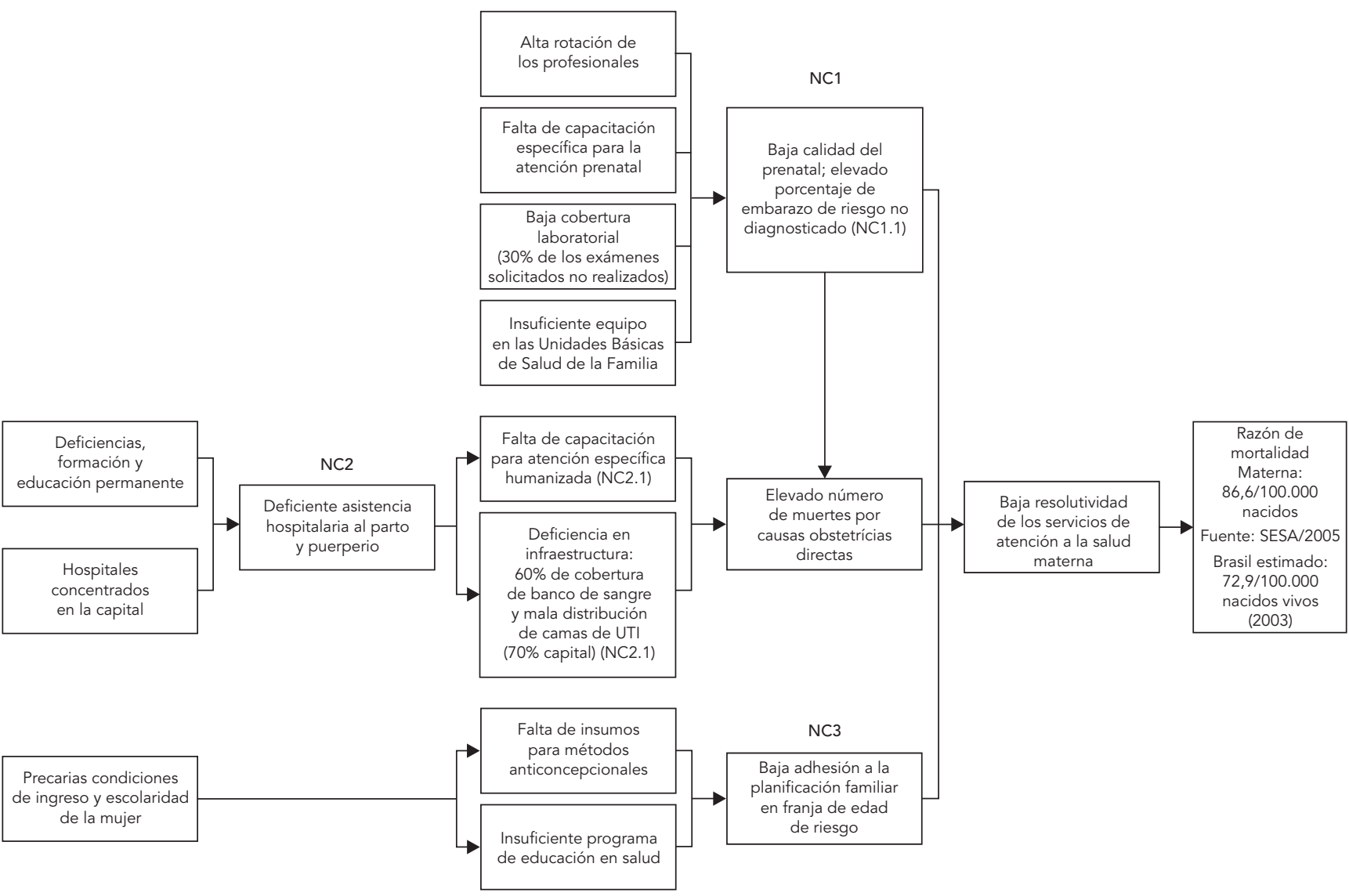

NC1

más precisos son los problemas de capacitación del personal que atiende (NC2.1) y los déficits de infraestructura: baja cobertura del banco de sangre y alta concentración de lechos de la unidad de tratamiento intensivo (UTI) en la capital, Fortaleza, con la consecuente desprotección del Estado como un todo (NC2.2).

- Línea de las insuficiencias relativas a la educación en salud y al programa correlacionado de planificación familiar (NC3.1).

Queda claro en el ejemplo que el alto número de muertes por problemas obstétricos es un indicador de baja capacidad de resolución, que deriva simultáneamente de la baja calidad de la atención primaria, de la deficiente asistencia hospitalaria y también del mal funcionamiento del programa de planificación familiar. Esto significa que las tres cadenas de causalidad o de argumentos causales se articulan horizontalmente produciendo un enunciado que expresa el punto de vista de la baja capacidad de resolución. Por lo tanto, aquí reconocemos un claro argumento coordinado. No obstante, es importante notar que cada subred de causalidad es un conjunto de argumentos subordinados, en la que - por ejemplo - la baja calidad de la atención primaria se explica por la rotación de los profesionales, la insuficiente disponibilidad de equipos en las 
Unidades del Programa de Salud Familiar (PSF), la baja cobertura del laboratorio y los problemas relativos a la capacitación específica del personal médico. En este caso estos problemas se coordinan también para generar una baja calidad, en la medida en que, por ejemplo, la resolución de un subproblema que corresponde a un argumento subordinado (laboratorio como causa de la baja calidad) no es garantía de que el problema derivado vaya a ser resuelto.

Otra posibilidad de aplicación de la teoría argumentativa se refiere a los esquemas argumentativos. Creemos que el uso de los esquemas argumentativos puede colaborar en buena medida para responder a las inquietudes de Matus, referentes a la cuestión de la vulnerabilidad de los argumentos del plan y de la solidez de las relaciones entre argumentos o causas. Entendemos por esquema argumentativo una manera más o menos convenida de representar la relación entre lo que se afirma en el argumento y lo que se afirma en el punto de vista 6 , o el modo específico en que las premisas llevan a la conclusión 18 . Los esquemas son patrones de argumentación que permiten identificar y evaluar tipos de argumentos corrientes. El esquema argumentativo predominante en la explicación del PES es el argumento de causa a efecto, que corresponde al tipo de relación que se establece entre los argumentos causales y entre estos y los descriptores del problema. En el primer caso, una causa derivada puede ser asumida como un punto de vista relativo a la causa que la genera o explica. Este esquema causal se transforma en el momento normativo en un esquema de medios y fines. Podemos reconocer en el momento explicativo incluso un tipo de argumentación sintomática, o argumento del signo, relativo al trabajo de descripción del problema de base y también de los nudos críticos. En la argumentación basada en una relación sintomática, un punto de vista es defendido citando en la discusión cierto signo, síntoma o marca distintiva de cuál es la conclusión del punto de vista. Sobre la base de esta relación de concomitancia, el hablante concluye que el punto de vista debe ser aceptado. 21 .

La validez o solidez de la argumentación dependería del tipo de esquema argumentativo escogido, como de la forma o del grado de corrección que caracteriza el uso de un determinado esquema. Para evaluar si cada esquema está siendo bien utilizado es necesario considerar ciertos elementos críticos, representados por preguntas específicas emparejadas a cada esquema. Se considera que estas preguntas serían algo así como reacciones críticas suscitadas por un antagonista en un proceso crítico, dialéctico, de discusión orientado al convencimiento. Así, van Eemeren ${ }^{6}$ sostiene que estas reacciones críticas constituyen un test completo para examinar la validez de una argumentación del tipo en cuestión.

En el caso de la argumentación sintomática o del argumento del signo, las cuestiones críticas serían 18:

CQ1: ¿Cuál es la fuerza de la correlación del signo con el evento significado?

CQ2: ¿Hay otros eventos que podrían ser descritos de manera más segura por el signo?

En el caso del argumento de causa a efecto, Walton et al. ${ }^{20}$ y Marraud 18 señalan las siguientes cuestiones críticas (agrupadas):

CQ1: ¿Qué razones hay para creer que los eventos de tipo E causan eventos de tipo E'? ¿Cómo es de fuerte la generalización causal? ¿Es la relación causal absolutamente verdadera?

CQ2: ¿Qué razones hay para creer que en la situación S se da un evento particular y de tipo E? ¿Si hay alguna evidencia, es la evidencia citada lo suficientemente fuerte para garantizar la generalización establecida?

CQ3: ¿Hay en la situación S otros factores que pueden interferir con la producción del efecto o contrarrestar el efecto en este caso?

En el ejemplo que anexamos, este último test implica la necesidad de tomar las tres redes de causalidad y formular las preguntas relativas al argumento de causa y efecto. Para ilustrarlo con la red de la asistencia hospitalaria, tendríamos que investigar las razones que explican el alto número de muertes obstétricas en el Estado de Ceará, en qué medida esta relación causal es lo suficientemente fuerte, cuál es la evidencia existente sobre una situación crítica de la asistencia y sobre su impacto, cuál es el grado de suficiencia de esta relación, o sea, si es la única fuente de mortalidad o hay otras, etc. Esto implica en buscar las evidencias sobre deficiencias de infraestructura, concentración de camas de terapia intensiva en la capital, distribución de la mortalidad en el interior del estado (donde el déficit de infraestructura sería más patente), sobre niveles de formación profesional, etc., es decir, evidencias sobre las razones argüidas para explicar la mortalidad. También significa investigar si no hay otros factores que interfieren en el efecto estudiado, como por ejemplo, problemas relacionados con otras formas de atención, lo que permite evaluar el grado de suficiencia de esta línea explicativa. En este caso, la asistencia hospitalaria parece no ser suficiente para explicar el efecto señalado, lo que habla a favor de una estructura argumentativa coordinada. Por lo tanto, podemos afirmar que la aplicación de este test sobre la adecuación de un esquema argumental estaría también vinculada al diseño de una determinada estructura argumentativa. Podría suceder, eventualmente, que 
no se encuentre evidencia sobre una mortalidad superior en zonas del interior, donde se verificó una mayor deficiencia de infraestructura. Esto puede significar un debilitamiento de la relación causal aludida, un comprometimiento de su consistencia, lo que obligaría a pensar en otras relaciones de mayor consistencia eventual.

Es importante hacer algunos comentarios finales sobre el esquema argumentativo de causalidad. Un concepto que nos parece fundamental es el de argumento de causalidad "derrotable". Pearl 22 y Walton et al. ${ }^{20}$ sostienen que la mayor parte de las aserciones causales en el lenguaje natural están sujetas a excepciones, significando que pueden ser rebatidas cuando nueva información se incorpora. Estas aserciones causales no podrían ser tratadas como generalizaciones universales o como relaciones condicionales típicas de un enfoque lógico-deductivo. Para Walton et al. 20 la causación no puede ser definida objetivamente. La causalidad no puede ser vista como una suerte de conexión absoluta o lógicamente necesaria entre pares de eventos. En este sentido, sería más adecuado pensar en relaciones causales dotadas de un determinado grado de probabilidad o plausibilidad. En esta misma línea, Matus 10 señala que toda argumentación causal implica una relación entre una causa y un efecto, mediada por circunstancias de contexto, de poca gobernabilidad, cambiantes, de difícil previsión. Esto sería típico de problemas mal estructurados. Así, la relación entre asistencia hospitalaria y mortalidad materna (del caso aquí presentado) dependería del nivel de articulación de la red hospitalaria (involucrando maternidades, hospitales más complejos y unidades de urgencia), del presupuesto sanitario y específicamente del volumen de financiación de la atención hospitalaria, del nivel de crecimiento económico, del grado de desarrollo regional, de los niveles de acceso geográfico a la atención, etc., es decir, sostener que una crisis de infraestructura hospitalaria genera más mortalidad materna es una aserción aceptable dentro de un determinado contexto que tiene que ser precisado, configurando una relación contingente, no necesaria en términos absolutos. De esta manera, podemos establecer una relación entre argumento causal "derrotable" y naturaleza mal estructurada de la problemática social de un plan situacional en salud.

Otro aspecto que nos parece importante es la definición de causalidad hecha en filosofía en términos de lo que serían condiciones necesarias y suficientes. En algunos casos de raciocinio causal, la causa es vista como condición necesaria, en otros como suficiente. La definición de necesidad no correspondería a la definición lógica, sino a un tipo de necesidad contingente o relativa, aplicable a acciones humanas o a eventos naturales. Para Walton et al. 20 el enfoque predominante en la actualidad consiste en considerar en conjunto condiciones necesarias y suficientes. Scriven 23 y Walton et al. 20 señalan que la producción de un efecto o resultado depende en general de una serie de condiciones necesarias en las que el conjunto pueda ser considerado suficiente. El modelo PES, que analizamos, se inscribe dentro de esta lógica cuando sostiene que la validez del VDP de un problema depende de que los descriptores sean necesarios y el conjunto suficiente. De la misma forma, podemos afirmar que un modelo causal válido o aceptable en planificación situacional es aquel en que los diferentes nudos críticos de una explicación son necesarios y el conjunto suficiente (para explicar un problema tal como se ha enunciado y descrito).

El análisis de validez de los esquemas argumentativos debe ser extendido al momento normativo del PES. En este momento se definen las operaciones del plan que enfrentarán los nudos críticos de cada problema. Uno de los instrumentos de cálculo de este momento consiste en una evaluación del impacto de todas las operaciones sobre cada uno de los nudos críticos, pues, aunque las operaciones sean diseñadas para enfrentar predominantemente un determinado nudo, ellas pueden impactar otros nudos en términos de impactos positivos o negativos y con una determinada intensidad. Esta evaluación puede ayudar a descartar operaciones que, a pesar de tener un impacto positivo sobre un nudo, pueden inviabilizar la confrontación de otros. Se debe considerar, por otro lado, que al atacar los signos o descriptores de los nudos críticos las operaciones pueden generar una dinámica de cambio que arrastra todos los nudos explicativos o argumentos subordinados de una misma cadena de causalidad, llevando a una modificación de los descriptores o signos del problema, que representan el efecto final de cada argumento de causa-efecto. En este momento se puede y se debe aplicar el conjunto de cuestiones críticas pertinentes para el argumento de medios-fines 18:

CQ1: ¿Qué razones hay para creer que eventos como c tienen consecuencias como b?

CQ2: i Es suficiente, en la situación dada, hacer c para producir el efecto $b$ ?

CQ3: iEs necesario, en la situación dada, hacer c para producir el efecto $b$ ?

CQ4: ¿Es factible c?

CQ5: ¿Realmente a quiere que b sea el caso? ¿Qué otros fines persigue a y cuales son prioritarios?

CQ5: ¿Qué otras consecuencias tendría c? ¿Estamos dispuestos a aceptar todas esas consecuencias para lograr $b$ ?

CQ7: Tomando en consideración todas las con- 
secuencias, favorables y desfavorables, de los distintos modos de lograr b, ¿cuál de ellos es preferible?

Sostenemos que el análisis de consecuencias favorables y desfavorables de este test coincide con el tipo de balance de impactos positivos y negativos de las operaciones, propuesto por el PES para la selección definitiva de los contenidos argumentales del plan. Según este balance, una determinada operación podría eventualmente ser descartada por contrarrestar el efecto de otra sobre un determinado nudo. Para mantener una operación la suma de sus efectos favorables debería superar significativamente eventuales efectos desfavorables. Terminamos aquí esta parte, planteando que otro tipo de análisis puede tener una importante utilidad en el proceso de la planificación situacional, además del análisis del grado de necesidad y suficiencia de los descriptores del problema y de los nudos - a que se hizo referencia anteriormente - corresponde al análisis del grado de necesidad y suficiencia de las operaciones del plan, así como a los compromisos que generan el efecto de transformar positivamente los descriptores o signos, el estudio del esquema argumentativo de medios a fines que ayudaría a realizar ese análisis.

\section{Conclusión}

Las principales distinciones y definiciones metodológicas del PES corresponden a expresiones lingüísticas. Estas expresiones pueden ser catalogadas en sus diferentes momentos de cálculo, según la teoría de los actos de habla de Austin y Searle. Cada un de estos actos cumple una función gerencial clara y contribuye en el logro del objetivo central de un plan situacional, sea cual sea el desarrollo de compromisos de acción.

El plan situacional es una apuesta argumentativa. De cara a problemas poco estructurados, el plan construye su aceptabilidad social y su confiabilidad en el marco de procesos argumentativos que se aproximan a un modelo crítico de discusión, de naturaleza dialéctica, donde varios actores se involucran directa o indirectamente en sus procesos de cálculo, buscando el convencimiento. Sin esta perspectiva, el plan deja de operar con los necesarios criterios de validez de sus expresiones, criterios que le permiten compensar la fuerte incertidumbre de esos procesos.

La teoría de la argumentación puede ayudar a explorar de manera más competente los esquemas argumentativos presentes en el diseño de un plan y a definir mejor la particular estructura argumentativa del conjunto de enunciados explicativos del mismo. Esta colaboración entre argumentación y planificación implica un desafío lógico y dialéctico, como el de construir argumentos explicativos y normativos necesarios y suficientes que le otorguen validez al plan y el de construir la base de relevancia y de aceptabilidad de sus premisas en el seno de un discusión crítica fundamentada, para de este modo poder alcanzar un punto de vista legítimo y eficaz. 
Resumen

Este trabajo pretende analizar el proceso de producción de un plan situacional, de acuerdo con una obra de referencia de la filosofía del lenguaje y teoría argumentativa. El enfoque básico en análisis fue desarrollado por Carlos Matus. Específicamente, se busca identificar la estructura y los esquemas argumentativos inherentes a la explicación situacional y al diseño normativo de las operaciones de un plan, asumiendo como parámetros de análisis los enfoques argumentativos de la pragma-dialéctica y de la lógica informal. Se utiliza un caso de explicación de un problema de salud para ilustrar este estudio. Metodológicamente, el estudio se apoya en la revisión bibliográfica sobre el asunto en cuestión y en el análisis de casos. Como resultado, el trabajo apunta al planteamiento de que el uso de las referencias apuntadas significa introducir un mayor rigor en el análisis de la validez de los argumentos causales y en el diseño de las propuestas de intervención, de tal manera que éstas pueden ser más concluyentes para el logro de los objetivos de un plan.

Planificación Estratégica; Planificación en Salud; Técnicos de Planificación
Agradecimientos

Al CAPES (Coordinación de Perfeccionamiento de Personal de Nivel Superior).

\section{Referencias}

1. Rivera FJU. Agir comunicativo e planejamento social: uma crítica ao enfoque estratégico. Rio de Janeiro: Editora Fiocruz; 1995.

2. Habermas J. Teoría de la acción comunicativa. Madrid: Taurus; 1989.

3. Flores F. La empresa del siglo XXI. Santiago: Hataché; 1989.

4. Austin J. How to do things with words. Oxford: Clarendon; 1962.

5. Searle JR. A taxonomy of illocucionary acts in Language, mind and knowledge. Minneapolis: University of Minnesota Press; 1976.

6. Van Eemeren FHV, Grootendorst R. Argumentación, comunicación y falacias. Santiago: Universidad Católica; 2006.

7. Matus C. Política, planejamento e governo. Brasília: Instituto de Pesquisa Econômica Aplicada; 1993.
8. Searle JR, Vanderveken D. Foundations of illocutionary logic. Cambridge: Cambridge University Press; 1985.

9. Rivera FJU, Artmann E. Planejamento e gestão em saúde: histórico e tendências com base numa visão comunicativa. Ciênc Saúde Coletiva 2010; 15:2265-74

10. Matus C. O plano como aposta. São Paulo Perspect 1991; 5:28-42.

11. Matus M. A teoria do jogo social. São Paulo: Fundação do Desenvolvimento Administrativo; 2005.

12. Rivera FJU, Artmann E. Planejamento e gestão em saúde: flexibilidade metodológica e agir comunicativo. Ciênc Saúde Coletiva 1999; 4:355-65.

13. Pitta AMR, Rivera FJU. Sobre pontos de partida: planejamento em comunicação e integralidade da atenção em saúde. Interface Comun Saúde Educ $2006 ; 10: 395-410$ 
14. Mitroff I. The subjective side of science. New York: American Elsevier; 1974.

15. Artmann E. O Planejamento Estratégico Situacional no nível local: um instrumento a favor de uma visão multissetorial. Rio de Janeiro: Instituto $\mathrm{Al}$ berto Luiz Coimbra de Pós-graduação e Pesquisa de Engenharia, Universidade Federal do Rio de Janeiro; 2000. (Cadernos da Oficina Social, 3. Desenvolvimento Local).

16. Toulmin S. Os usos do argumento. São Paulo: Editora Martins Fontes; 2001.

17. Ortega y Gasset J. Historia como sistema. Madrid: Espasa-Calpe; 1971.

18. Marraud H. Methodus argumentandi. Madrid: Universidad Autónoma de Madrid; 2007.
19. Sagüillo JM. El Arte de persuadir. La Coruña: Ludus; 2000.

20. Walton D, Reed C, Macagno F. Argumentation schemes. Cambridge: Cambridge University Press; 2008.

21. Van Eemeren FHV, Grootendorst R, Henkemans FS. Argumentación: análisis, evaluación, presentación. Buenos Aires: Biblos; 2006.

22. Pearl J. Causality: models, reasoning and inference. Cambridge: Cambridge University Press; 2000.

23. Scriven M. Reasoning. New York: McGraw-Hill; 1977.

Recibido el 14/Mar/2011

Versión final presentada el 03/Jun/2011

Aprobado el 22/Jun/2011 